8 Evgeny Andronov ${ }^{1}$

13 Institute for Agricultural Microbiology,, Saint-Petersburg, Russia

$16 *$ Corresponding author

\title{
19 Abstract
}

The rhizosphere community represents an “ecological interface” between plant and soil,

21 providing the plant with a number of advantages. Close connection and mutual influence in this 
22 communication allow to talk about the self-adjusting “plant-rhizosphere community” system, which

23 should be be studied in connection. Diversity estimation is one of the ways of describing both

24 bacterial and plant communities. Based on the literature, there are two assumptions of how the

25 diversity of plant communities related to the diversity of bacterial communities: 1) an increase in

26 the species richness of plants leads to an increase in the number of available micro-niches, and

27 increasing of microbial diversity, 2) an increase in the species richness of plants is accompanied by

28 the predominant development of bacteria from highly productive specific taxa and decreasing in the

29 diversity of microorganisms. Experimental studies show controversial results.

We analyzed field sites (rye crop field and two fallow sites), using DNA isolation of both the

31 plant root mass (followed by sequencing of the ITS1 region) and rhizosphere microorganisms

32 (followed by sequencing of the 16s rDNA V4 region). This allowed us to 1) accurately determine

33 the abundance and taxonomic position of plant communities; 2) extract information about both

34 plant and microbial communities from the same sample.

There was no correlation between alpha-diversity indices of plants and rhizosphere

communities. Alpha-diversity connection should be explored in similar plant communities, such as

synusia. We hypothesize, that the significant differences in plant abundances lead to significant

\section{Introduction}

As the formation of a specific microbial community near the plant root, the phenomenon of

45 the rhizosphere effect has been the subject of many works of both classical and modern biology. 
46 The rhizosphere community represents an “ecological interface” between plant and soil, providing

47 the plant with a number of advantages such as growth stimulation, protection from pathogens,

48 nutrition, among others [1, 2]. The source of the rhizosphere microbiome is both the microbial

49 community of plant seeds [3] and the community of soil microorganisms [4]. The composition and

50 abundance of plant root exudates determine the formation of the bacterial community $[5,6]$. The

51 source of the microbiome, and the development of it, thus forms the final community.

Diversity in both sources and ways of development lead to the specificity of the rhizosphere

53 microbiome. Several factors can affect the composition of rhizosphere communities. In addition to

54 the type and agrochemical properties of the soil, the genotype of the plant (species [7, 8] and

55 cultivar [9]) is a significant factor for microbiome development. This can be explained by the

56 specific exudation spectrum from various plants, as the spectrum of secreted substances depends not

57 only on the species or cultivar but also on the developmental phase, physiological state, etc. [10,

58 11]. In turn, the microbial communities themselves affect the metabolic status of the plant [12],

59 which allows us to talk about the "plant-rhizosphere community" system as a self-adjusting system

60 (a kind of "gut-brain axis" in plants). This system is additionally complicated by the high diversity

61 of plant species, which is natural in indigenous plants populations.

Diversity estimation is one of the ways of describing both bacterial and plant communities. As

63 self-adjusting systems, rhizosphere microbiomes and plant communities are connected, and

64 therefore, the following question arises: is the diversity of plant communities related to the diversity

65 of bacterial communities? Based on the literature, there are two assumptions: 1) an increase in the

66 species richness of plants leads to an increase in the number of available micro-niches, which leads

67 to an increase in microbial diversity, 2) an increase in the species richness of plants is accompanied

68 by the predominant development of bacteria from highly productive specific taxa, which leads to a

69 general decrease in the diversity of microorganisms [13]. Experimental studies of this relationship

70 show controversial results: some studies indicate the absence or negative correlation between plant 
71 and bacterial richness $[14,15]$, whereas others show the presence of a positive relationship [16].

72 Beta diversity indices show an unambiguous positive correlation between the distances of plant and

73 bacterial communities [17].

There are examples of research of similar relationships between the diversity of plants and the associated microorganisms. A close relationship was demonstrated in a study of the diversity of the rhizobial soil community (by the nodA gene) and the diversity of their symbiotic hosts (the NFR5 and $\mathrm{K} 1$ genes of leguminous plants), allowing the authors to formulate the hypothesis of

78 "evolutionary molding", where the plant community plays the role of the rigid matrix and the

79 microbial community acts as a “molding” substance. The whole process not only links diversities,

80 but even phylogenetic topologies; for the description of the last phenomenon, the concept of beta-

81 topological diversity has been introduced [18]. Another example is the close relationship between

82 the diversity of Galega rhizobia and their two hosts, G. orientalis and G. officinales, revealed at the

83 level of genomic AFLP fingerprints [19]. However, for less closely integrated systems with

84 indigenous plant communities, analyzed by taxonomic rather than functional markers, such a

85 relationship has not yet been shown.

One of the reasons for the uncertainty in this area is, most likely, the problem of the correct

87 estimation of plant diversity, for which the same algorithms that are used today for the analysis of

88 microbial diversity could be applied. The development of NGS methods for the estimation of

89 microbial diversity, from DNA extraction methods to statistical analysis of libraries (16S, ITS), has

90 led to the rise of this area observed today [20]. However, in most papers, plant diversity is

91 determined using geobotanical methods $[13,15]$, but this approach is not accurate enough in both

92 determining the species in the communities and their abundances [21]. In addition, in the context of

93 the aim of this study, there is a question about the correspondence between the "aboveground" and

94 "belowground" plant diversities, which can be quite different [22]. 
Therefore, in this work, we analyzed the diversity of the plant population via direct DNA

isolation of the plant root mass, followed by NGS sequencing of the ITS1 region; rhizosphere soil

samples were taken from the same root sample. This allowed us to 1) accurately determine the approaches as used for the rhizosphere community of microorganisms. Thus, we were able to use the same algorithms and metrics to analyze both communities.

\section{Materials and Methods}

Samples were collected on July 21, 2017, on fields of the Pskov Research Institute of

Agricultural Sciences and Rodina State Farm in the Pskov region, Russia (coordinates of the collection point are 57.845611 N 28.201028 E). We select one site within a rye crop field (referred to as Monoculture Rye or MonoR) and two fallow sites outside the field border from two locations, dominated by cereals (Polyculture Cereal or PolyC) and Galium and Dactylis species (Polyculture description of the sampling sites is provided in the S1 Table and S1 Fig.

Bricks of topsoil (about 15 x 15 x $10 \mathrm{~cm}$ ) were collected and stored in individual packages not

112 longer than 48 hours. In the laboratory, bulk soil was gently removed manually by shaking, and 30

$113 \mathrm{~g}$ of the root mass was intensively shaken with $50 \mathrm{ml}$ of $0.005 \mathrm{M}$ Na-phosphate buffer in a Pulsifier

114 II (Microgen, UK) in provided bags (PUL512 Bags) for 1 min. The liquid fraction was centrifuged,

115 and the pellet was used to isolate the total rhizosphere DNA. The root mass was used to isolate

116 plant DNA.

Procaryotic DNA from the pellet was isolated using the MN NucleoSpin Soil Kit (Macherey-

118 Nagel, Germany) and a Precellus 24 homogenizer (Bertin, USA). Quality control was carried out by 
119 PCR and agarose gel electrophoresis. Sequencing of the V4 variable region of the 16S rRNA gene

120 was performed on an Illumina MiSEQ sequencer, using the primers F515

121 (GTGCCAGCMGCCGCGGTAA) and R806 (GGACTACVSGGGTATCTAAT) [23].

Plant DNA from roots was isolated using mechanical destruction in liquid nitrogen, followed

123 by phenol extraction; the quality of the DNA was also checked via agarose gel electrophoresis.

124 Sequencing of the ITS1 variable region was performed on an Illumina MiSEQ sequencer, using the

125 primers ITS-p5 (YGACTCTCGGCAACGGATA) and ITS-u2

126 (GCGTTCAAAGAYTCGATGRTTC) [24].

The general processing of sequences was carried out in R 3.6.4, using the dada2 (v. 1.14.1)

128 [25] and phyloseq (v. 1.30.0) [26]packages. For taxonomic annotation, the databases SILVA 138

129 [27] and PLANiTS [28] were used.

130 The main alpha- and beta-metrics were calculated using the phyloseq and picante [29]

131 packages. For the mean p-distance in a library, we used the home-brew script with following steps:

132 1) make multiple alignment for reference sequences; 2) extract p-value for every pair of sequence;

133 3) multiple this p-value to abundance of both seqences; 3) sum all values. Correlations between

134 diversity indices were calculated using Spearman correlation. Significant differences in abundances

135 of taxa between sites were determined using theDeSEQ2 package [30].

137 https://www.ncbi.nlm.nih.gov/sra/PRJNA649486.

\section{Results}

140 The ITS1 sequencing of plant DNA yielded 230 ASVs. The taxonomic position at genus level

141 was defined for 217 of them; the number of reads per sample after rarefaction was 14,210.

142 Regarding 16s rDNA, we found 5,284 ASVs, with 15,487 reads per sample. 
Fig 1 shows the taxonomic composition of the communities. The geobotanic description

144 (provided in the S1 Table) corresponded with the composition structure according to ITS1

145 sequencing (Fig 1A). Almost all reads from MonoR libraries were attributed as Secale; PolyC

146 (samples from the cereal synusia) had about half the reads from Poa, followed by Elymus and

147 Dactilus. PolyG (samples from Galium and Dactylis synusia) was more diverse; most reads were

148 attributed as Galium, Poa. Despite the geobotanical description of this synusia as mixed Galium

149 and Dactylis, there was no evidence of a great amount of Dactylis in the PolyG libraries, which can

150 be explained by difficulties in describing the graminae vegetation outside the flowering phase.

\section{Fig 1. Relative abundances of plant and bacterial communities in the experimental fields}

The bacterial communities from the samples (Fig 1B) were typical for rhizosphere

microbiomes [11]. The communities from the two fallow sites (PolyC, PolyG) were similar,

whereas the communities from the rye site (MonoR) contained more Gammaproteobacteria

(Proteobacteria) and Bacteroidia (Bacteroideta). Differential abundance analysis allowed us to find

584 microbial taxa, which significantly differed between two sites, with information about

abundance in repeats. When comparing PolyC and PolyG, none of the OTUs was marked as

significantly different. The results of the pairwise comparison of PolyC and PolyG with MonoR are

159 shown in Fig 2 at the order level.

Fig 2. Mean abundances of bacterial orders with different inter-source abundances

(according to DeSEQ2)

In this comparison, the most abundant OTUs in rye crop rhizosphere microbiomes were from

163 Proteobacteria (orders Pseudomonadales, Sphingomonadales, Enterobacteriales), followed by

164 Armatimonadota (Fimbriimonadales), Acidobacteriales (Bryobacterales), and Actinobacteriota

165 (Micrococcales). In fallow root microbiomes, Verrucomicrobia (Chthoniobacteriales),

166 Acidobacteria (Blastocatellales), and Actinobacteria (Pseudonocardiales) were more abundant. 
For all samples, the most common alpha-diversity indices, observed OTUs, Shannon,

168 Simpson, mpd (mean pairwise distance), and p-dist (mean p-distance, restored from alignment, see

169 Materials and Methods), were calculated at different taxonomic levels (Fig 3).

Fig 3. Alpha-diversity indices for plant and bacterial communities (significance for

ANOVA in groups; ns - non-significant, * $-\mathbf{p}<0.05, * * \mathbf{p}<0.01, * * * \mathbf{p}<0.005)$

The plant communities were highly diverse at different levels and indices (Fig 3A). The

observed OTU index was not useful because of the presence of weedy plants in the rye crop.

174 Weighted indices, such as mpd or p-dist, were more suitable; the differences between sample sites

were significant for both indices up to the order level.

The bacterial communities were less diverse. The observed OTUs as well as the p-dist and

mpd indices showed that communities from the PolyC site were more diverse, mostly at low

taxonomical levels (OTUs, genus). Interestingly, samples from rye roots (MonoR) were

significantly less diverse at phylum level, whereas at other taxonomical ranks, there were no such differences.

For each index on the OTU taxonomic level, the correlation between plant diversity indices

and microbial diversity indices was calculated. We observed no significant correlations between plant and rhizosphere microbial diversity.

Fig 4 shows the beta-diversity indices (Bray distance and weighted UniFrac). According to the weighted UniFrac index (Dunn index for roots 0.316 , for microorganisms 0.821 ), the samples were mixed (Fig 4A). Samples from rhizosphere communities from PolyC and PolyG were mixed in one cluster. While the MonoR and PolyC samples were close, the PolyG samples were different. This might be connected to the phylogenetic composition of the population; Secale and Poa, the two 


\section{Fig 4. Beta-diversity indices for plant and bacterial communities}

According to the Bray distance (Dunn index for roots 0.832, for microorganisms 0.909), all

communities, from plants and from rhizospheres, formed their own clusters (Fig 3B).

The correlation between the distance matrix in plant and bacterial communities was high for the Bray distance $(\mathrm{R}=0.866, \mathrm{p}=0.01)$ and not significant for the weighted UniFrac index. Fig 5 shows the results of the beta-distance correlation, excluding the intra-cluster distance (distance between repeats of the same sample).

\section{Fig 5. Correlation between beta-diversity indices}

\section{Discussion}

The aim of this work was to determine the potential connection between the diversity of plant communities and the diversity of rhizosphere microbiomes. For this purpose, we used three sites with different plant communities (synusiae) in the same location: a rye crop monoculture (MonoR) and two polyculture fallow sites, dominated by cereals (PolyC) and Galium and Dactylis (PolyG).

Despite differences in crop species and tillage, soil type, water regime, and main soil properties were similar for all three sites, minimizing the influence of these factors on microbial communities.

The novel approach to estimate plant community composition (ITS1 sequencing) is highly effective. Almost all (217 of 230) determined ASVs were annotated, and the composition and abundance of plant genera fit the geobotanical description. In addition, this method allows 1) to microorganisms); 2) use the same sample for both plant and microbiome analysis (therefore, each rhizosphere library matches with the plant library); 3) use standard approaches for processing and calculating diversity indices and generating bar graphs. 
215 variation in the spectrum of exudates could be one of the possible mechanisms responsible for the

216 diversity of the rhizosphere microbial community. This spectrum is specific for various plant

217 species and genotypes, and therefore, in the case of the whole plant community, we can talk about

218 an "exudome" of the community, whose structure depends on the abundance of plants in the

219 community. This exudome can be described by the number of the individual plant spectra and by

220 their weights (amount of exudate spectra of exact plant species in all exxudome), according plant

221 abundances. In the comparison of two polycultures, the number of plant species is the same (as

222 shown in Fig 3A), with variations in abundance; it therefore seems logical that the exudomes of

223 these communities also will differ not in the number of different spectra but in their weights. In

224 contrast, when comparing monoculture plants communities with polyculture ones, there are

225 differences in the number of plant species. In this case, exudomes of plant communities will differ

226 in the number of different spectra and in their weights.

In this study, using modern approaches, we found no correlation between different alpha-

228 diversity indices. According to all indices, rhizosphere communities in the monoculture site

229 (MonoR) were as diverse as those in the polycultures (PolyR, PolyC), whereas the plant community

230 diversity in the monoculture was obviously lower. Regarding the polyculture (and mpd and p-dist

231 indices), more diverse plant communities (PolyG) showed a less diverse rhizosphere microbial

232 community. This might be evidence of a negative connection, as suggested by Goberna and

233 coauthors [13], but this connection can be found only in plant communities with close exudomes

234 (differing in abundance by not in the number of spectra). Significant differences in exudomes can

235 lead to different microbial response and a loss of similarity, when environmental factors override

236 the realistic relationship. Perhaps, this variation in exudome abundance allowed Schlatter and

237 colleagues to show that in artificial plant communities, there is a decrease in bacterial diversity with

238 increasing richness $(1,4,8,16$ species $)[31]$. 
Despite the expected main difference in plant diversity between poly- and monocultures, with

240 a moderate variation between polycultures, both alpha- and beta-diversity of the plants showed

241 significant variations between synusiae. In the community of different cereals, PolyG in most cases

242 is significantly more diverse by all alpha-diversity indices, and shows inter-sample variability

243 (sample PolyG.3). This effect is also clear at higher taxonomic levels (family or order).

Differential abundance analysis did not show any difference between rhizosphere

communities of polycultures. Similarly, OTUs and Shannon indices of these samples were also not recommended.

In contrary, when comparing the microbial communities of the polyculture sites with those of

251 the monoculture, there was a significant difference in the observed OTUs, whereas phylogeneticrelated indices (mpd, p-dist) showed no clear pattern. Differential abundance analysis enabled us to identify taxa with significant differences in relative diversity, most likely because of significant differences in exudome profiles and the loss of similarity between plant-microbial systems. mono- and polycultures were large (about $60 \%$ of the explained variance in PcoA plots). This 
The significant distance in beta-diversity is connected to the differences in the abundances of the different taxa. Hypothesized as a molding matrix, differences in abundances between plant communities are not useful, whereas abundances of microbial taxa represent the specificity of are typical for plant rhizosphere communities [32], and Fimbriimonadales has previously been described as a bacterial taxon from the Anthurium andraeanum rhizosphere community [33]. In the same case, Blastocatellales has previously been described as an oligotrophic, slightly acidophilic to neutrophilic mesophile from arid soils [34]. However, these data are controversial, as they can

272 describe a novel location of this microorganism or a biased database. More precise data can be

273 obtained by functional or full-genomic analyses of rhizosphere communities.

Despite the absence of a clear correlation between plant and bacterial diversity, comparing the results with the previously mentioned concept of evolutionary molding, where the plant population tighter than the rhizosphere community interaction; 2) in this work, we used regular taxonomic marker genes, not specific plant or bacterial genes. A closer interaction could be revealed with the use of genes directly involved in plant-rhizosphere communication processes. This could be plant genes involved in plant root exudation and responses to bacterial signals (for example, 


\section{Conclusions}

1. Sequencing of the ITS1 region is highly effective for the taxonomical annotation of plant communities. Almost all ASVs were attributed; abundances of taxa fairly corresponded to the geobotanical descriptions.

2. There was no correlation between alpha-diversity indices of plants and rhizosphere communities. Alpha-diversity connection should be explored in similar plant communities, such as synusia. Significant differences in plant abundances lead to significant changes in exudation profiles, different microbial responses, and the loss of diversity connection.

3. In contrast, the beta-diversity between rhizosphere communities and plant communities is

potential correlation (as reported in the literature) or by the presence of statistical artifacts.

\section{Acknowledgments}

This work was performed using the equipment of the Genome Technologies and Cell Biology 


\section{References}

1. Gaiero JR, McCall CA, Thompson KA, Day NJ, Best AS, Dunfield KE. 2013. Inside the root microbiome: bacterial root endophytes and plant growth promotion. American Journal of

2. Philippot L, Raaijmakers JM, Lemanceau P, van der Putten WH. Going back to the roots: the microbial ecology of the rhizosphere. Nat Rev Microbiol. 2013 Nov;11(11):789-99. doi: doi: 10.1111/1758-2229.12760. Epub 2019 May 21. PMID: 31054200. doi: 10.1111/j.1574-6941.2009.00654.x. Epub 2009 Feb 25. PMID: 19243436. 
Natl Acad Sci U S A. 2015 Feb 24;112(8):E911-20. doi: 10.1073/pnas.1414592112. Epub 2015 Jan

328

329

330

331

332

333

334
20. PMID: 25605935; PMCID: PMC4345613.

9. Lundberg DS, Lebeis SL, Paredes SH, Yourstone S, Gehring J, Malfatti S, Tremblay J,

Engelbrektson A, Kunin V, Del Rio TG, Edgar RC, Eickhorst T, Ley RE, Hugenholtz P, Tringe SG,

Dangl JL. Defining the core Arabidopsis thaliana root microbiome. Nature. 2012 Aug

2;488(7409):86-90. doi: 10.1038/nature11237. PMID: 22859206; PMCID: PMC4074413.

10. Sasse J, Martinoia E, Northen T. Feed Your Friends: Do Plant Exudates Shape the Root

Microbiome? Trends Plant Sci. 2018 Jan;23(1):25-41. doi: 10.1016/j.tplants.2017.09.003. Epub

2017 Oct 17. PMID: 29050989.

11. Zverev A, Pershina E, Shapkin V, Kichko A, Mitrofanova O, Kobylyanskii V, Yuzikhin

O, Belimov A, Andronov E. Molecular Analysis of the Rhizosphere Microbial Communities from

Gramineous Plants Grown on Contrasting Soils. Microbiology. 2020. 89. 231-241.

10.1134/S002626172001018X.

12. Vives-Peris V, de Ollas C, Gómez-Cadenas A, Pérez-Clemente RM. Root exudates: from plant to rhizosphere and beyond. Plant Cell Rep. 2020 Jan;39(1):3-17. doi: 10.1007/s00299-01902447-5. Epub 2019 Jul 25. PMID: 31346716.

13. Goberna M, Navarro-Cano JA, Verdú M. Opposing phylogenetic diversity gradients of plant and soil bacterial communities. Proc Biol Sci. 2016 Feb 24;283(1825):20153003. doi: 10.1098/rspb.2015.3003. PMID: 26888037; PMCID: PMC4810838.

14. Dassen S, Cortois R, Martens H, de Hollander M, Kowalchuk GA, van der Putten WH, De Deyn GB. Differential responses of soil bacteria, fungi, archaea and protists to plant species richness and plant functional group identity. Mol Ecol. 2017 Aug;26(15):4085-4098. doi: 10.1111/mec.14175. Epub 2017 Jun 2. PMID: 28489329. 
15. Li H, Wang X, Liang C, Hao Z, Zhou L, Ma S, Li X, Yang S, Yao F, Jiang Y.

351

352

353

Aboveground-belowground biodiversity linkages differ in early and late successional temperate forests. Sci Rep. 2015 Jul 17;5:12234. doi: 10.1038/srep12234. PMID: 26184121; PMCID:

PMC4505317.

\section{Sun YQ, Wang J, Shen C, He JZ, Ge Y. Plant evenness modulates the effect of plant} richness on soil bacterial diversity. Sci Total Environ. 2019 Apr 20;662:8-14. doi:

10.1016/j.scitotenv.2019.01.211. Epub 2019 Jan 18. PMID: 30682712.

17. Prober SM, Leff JW, Bates ST, Borer ET, Firn J, Harpole WS, Lind EM, Seabloom EW, Adler PB, Bakker JD, Cleland EE, DeCrappeo NM, DeLorenze E, Hagenah N, Hautier Y, Hofmockel KS, Kirkman KP, Knops JM, La Pierre KJ, MacDougall AS, McCulley RL, Mitchell CE, Risch AC, Schuetz M, Stevens CJ, Williams RJ, Fierer N. Plant diversity predicts beta but not alpha diversity of soil microbes across grasslands worldwide. Ecol Lett. 2015 Jan;18(1):85-95.

18. Igolkina AA, Porozov YB, Chizhevskaya EP, Andronov EE. Structural Insight Into the Role of Mutual Polymorphism and Conservatism in the Contact Zone of the NFR5-K1 Heterodimer With the Nod Factor. Front Plant Sci. 2018 Apr 11;9:344. doi: 10.3389/fpls.2018.00344. PMID: $29706972 ;$ PMCID: PMC5909492.

19. Osterman J, Chizhevskaja EP, Andronov EE, Fewer DP, Terefework Z, Roumiantseva ML, Onichtchouk OP, Dresler-Nurmi A, Simarov BV, Dzyubenko NI, Lindström K. Galega orientalis is more diverse than Galega officinalis in Caucasus--whole-genome AFLP analysis and phylogenetics of symbiosis-related genes. Mol Ecol. 2011 Nov;20(22):4808-21. doi: 10.1111/j.1365-294X.2011.05291.x. Epub 2011 Oct 10. PMID: 21980996.

20. Sharma TR, Devanna BN, Kiran K, Singh PK, Arora K, Jain P, Tiwari IM, Dubey H, Saklani B, Kumari M, Singh J, Jaswal R, Kapoor R, Pawar DV, Sinha S, Bisht DS, Solanke AU, Mondal TK. Status and Prospects of Next Generation Sequencing Technologies in Crop Plants. 
374 Curr Issues Mol Biol. 2018;27:1-36. doi: 10.21775/cimb.027.001. Epub 2017 Sep 8. PMID:

37528885172.

21. Parker J, Helmstetter AJ, Devey D, Wilkinson T, Papadopulos AST. Field-based species

identification of closely-related plants using real-time nanopore sequencing. Sci Rep. 2017 Aug

21;7(1):8345. doi: 10.1038/s41598-017-08461-5. PMID: 28827531; PMCID: PMC5566789.

22. Skuodiene R., Tomchuk D. Root mass and root to shoot ratio of different perennial forage

plants under western Lithuania climatic conditions. 2015 Romanian Agricultural Research. 1-11.

Fierer N, Knight R. 2011. Global patterns of 16S rRNA diversity at a depth of millions of

sequences per sample. Proc Natl Acad Sci U S A 108(Suppl):4516-4522.

doi:10.1073/pnas.1000080107.

24. Cheng T, Xu C, Lei L, Li C, Zhang Y, Zhou S. Barcoding the kingdom Plantae: new PCR

primers for ITS regions of plants with improved universality and specificity. Mol Ecol Resour.

2016 Jan;16(1):138-49. doi: 10.1111/1755-0998.12438. Epub 2015 Jul 3. PMID: 26084789.

25. Callahan BJ, McMurdie PJ, Rosen MJ, Han AW, Johnson AJ, Holmes SP. DADA2: High-

resolution sample inference from Illumina amplicon data. Nat Methods. 2016 Jul;13(7):581-3. doi:

10.1038/nmeth.3869. Epub 2016 May 23. PMID: 27214047; PMCID: PMC4927377.

26. McMurdie PJ, Holmes S. phyloseq: an R package for reproducible interactive analysis and

graphics of microbiome census data. PLoS One. 2013 Apr 22;8(4):e61217. doi:

393

10.1371/journal.pone.0061217. PMID: 23630581; PMCID: PMC3632530. 
28. Banchi E, Ametrano CG, Greco S, Stanković D, Muggia L, Pallavicini A. PLANiTS: a

399

400

401

402

403

404

405

406

407

408

409

410

411

412

curated sequence reference dataset for plant ITS DNA metabarcoding. Database (Oxford). 2020 Jan 1;2020:baz155. doi: 10.1093/database/baz155. PMID: 32016319; PMCID: PMC6997939.

29. Kembel SW, Cowan PD, Helmus MR, Cornwell WK, Morlon H, Ackerly DD, Blomberg SP, Webb CO. Picante: R tools for integrating phylogenies and ecology. Bioinformatics. 2010 Jun 1;26(11):1463-4. doi: 10.1093/bioinformatics/btq166. Epub 2010 Apr 15. PMID: 20395285.

30. Love MI, Huber W, Anders S. Moderated estimation of fold change and dispersion for RNA-seq data with DESeq2. Genome Biol. 2014;15(12):550. doi: 10.1186/s13059-014-0550-8. PMID: 25516281; PMCID: PMC4302049.

31. Schlatter DC, Bakker MG, Bradeen JM, Kinkel LL. Plant community richness and microbial interactions structure bacterial communities in soil. Ecology. 2015 Jan;96(1):134-42. doi: 10.1890/13-1648.1. PMID: 26236898.

32. Hartman K, van der Heijden MG, Roussely-Provent V, Walser JC, Schlaeppi K.

Deciphering composition and function of the root microbiome of a legume plant. Microbiome. 2017;5(1):2. Published 2017 Jan 17. doi:10.1186/s40168-016-0220-z

33. Sarria-Guzmán Y, Chávez-Romero Y, Gómez-Acata S, Montes-Molina JA, MoralesSalazar E, Dendooven L, Navarro-Noya YE. Bacterial Communities Associated with Different Anthurium andraeanum L. Plant Tissues. Microbes Environ. 2016 Sep 29;31(3):321-8. doi: 10.1264/jsme2.ME16099. Epub 2016 Aug 11. PMID: 27524305; PMCID: PMC5017810.

34. Pascual J, Wüst PK, Geppert A, Foesel BU, Huber KJ, Overmann J. Novel isolates double the number of chemotrophic species and allow the first description of higher taxa in Acidobacteria subdivision 4. Syst Appl Microbiol. 2015 Dec;38(8):534-44. doi: 10.1016/j.syapm.2015.08.001. Epub 2015 Sep 28. PMID: 26460220. 


\section{Supporting information}

S1 Fig. General view, current location and root samples. MonoR — rye crop field; PolyC -

424 forb and cereal meadow dominated by cereals ; PolyG - forb and cereal meadow dominated by

425 Galium and Dactylis species. Samples are: 1 - MonoR.1; 2 - MonoR.2; 3 - MonoR.3; 4 -

426 PolyG.1; 5 - PolyG.2; 6 - PolyG.3; 7 - PolyC.1; 8 - PolyC.2; 9 - PolyC.3; 


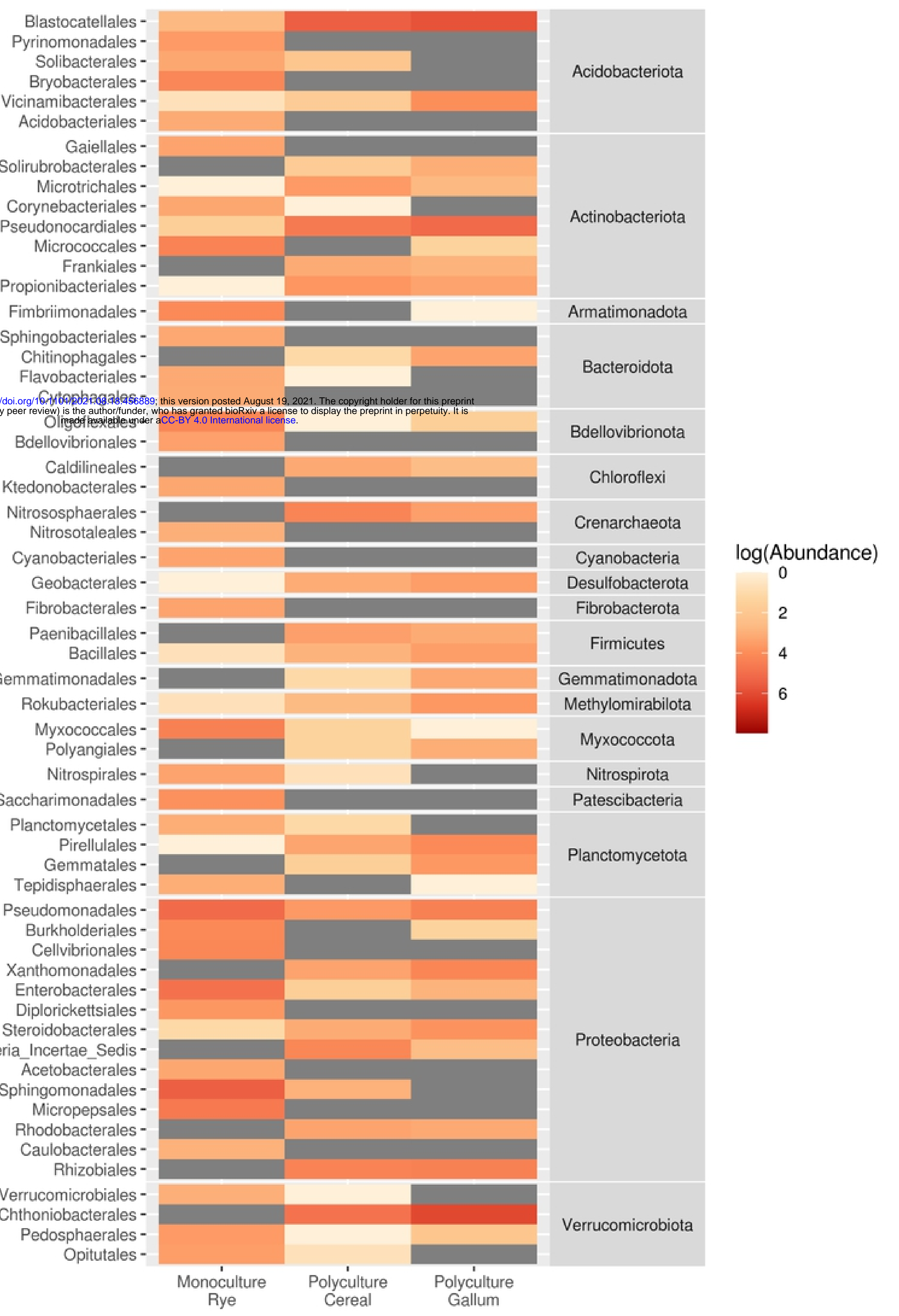


A. Weighted UniFrac

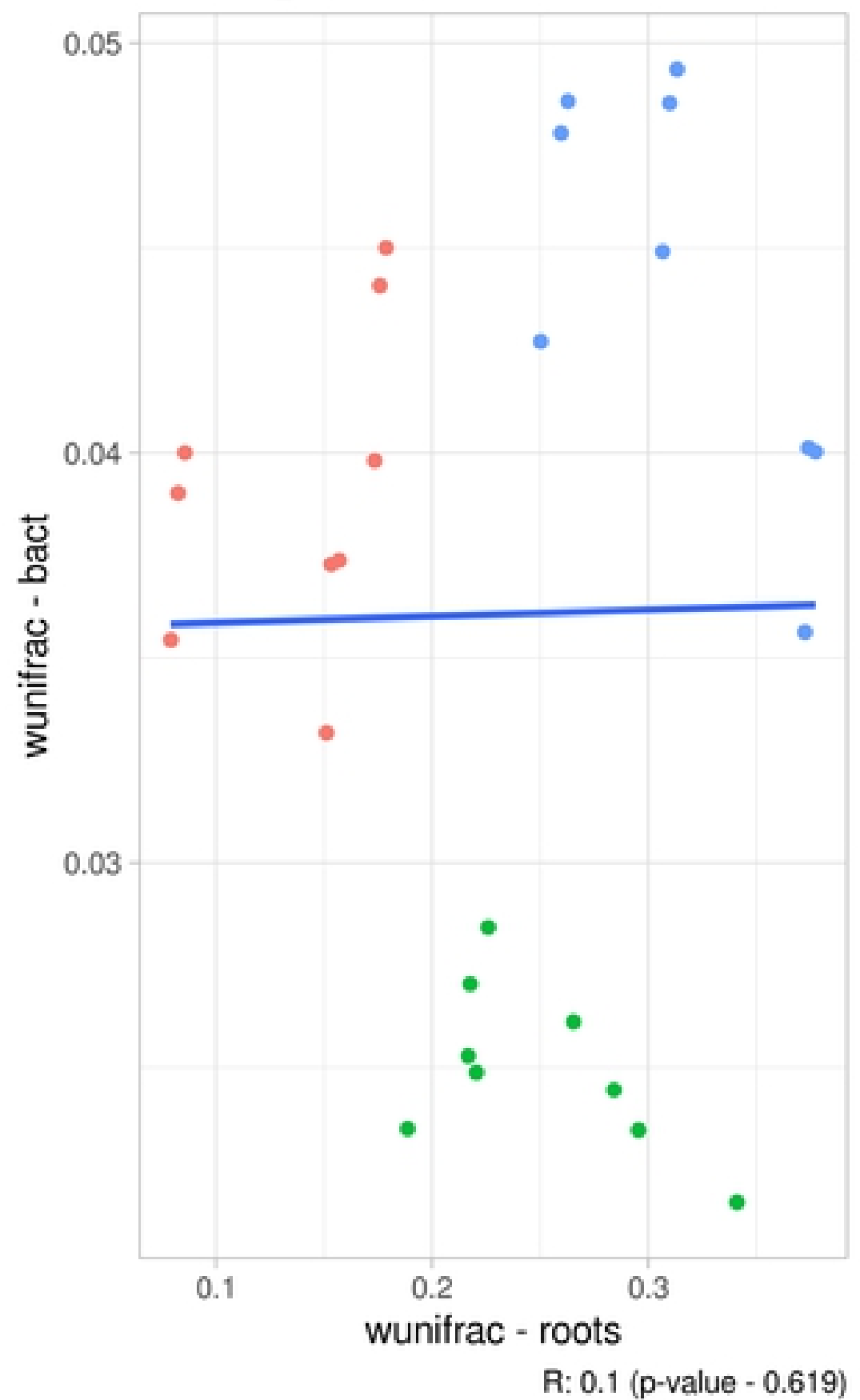

\section{B. Bray distance}

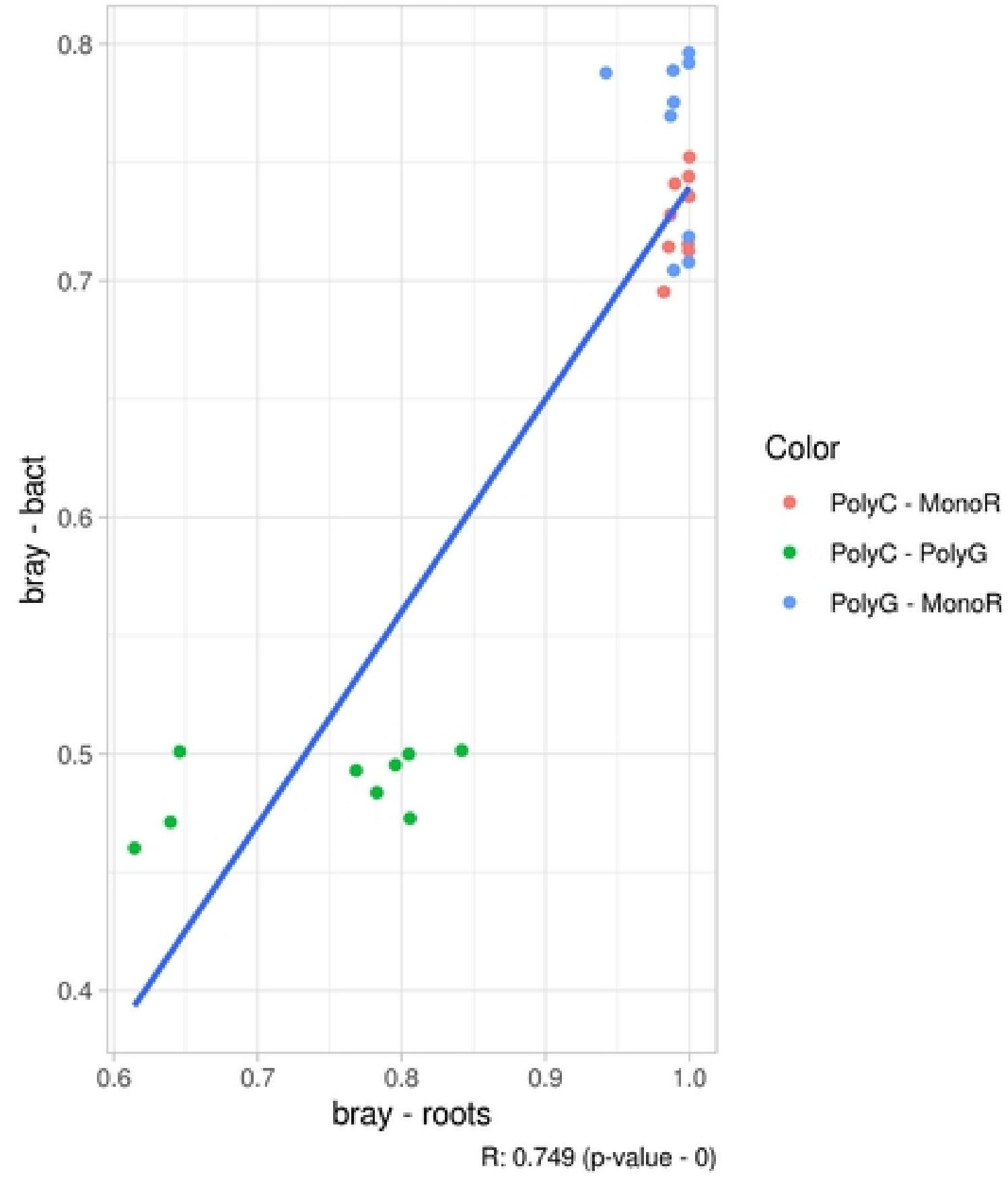

Fig 5 


\section{A. Plant diversity}

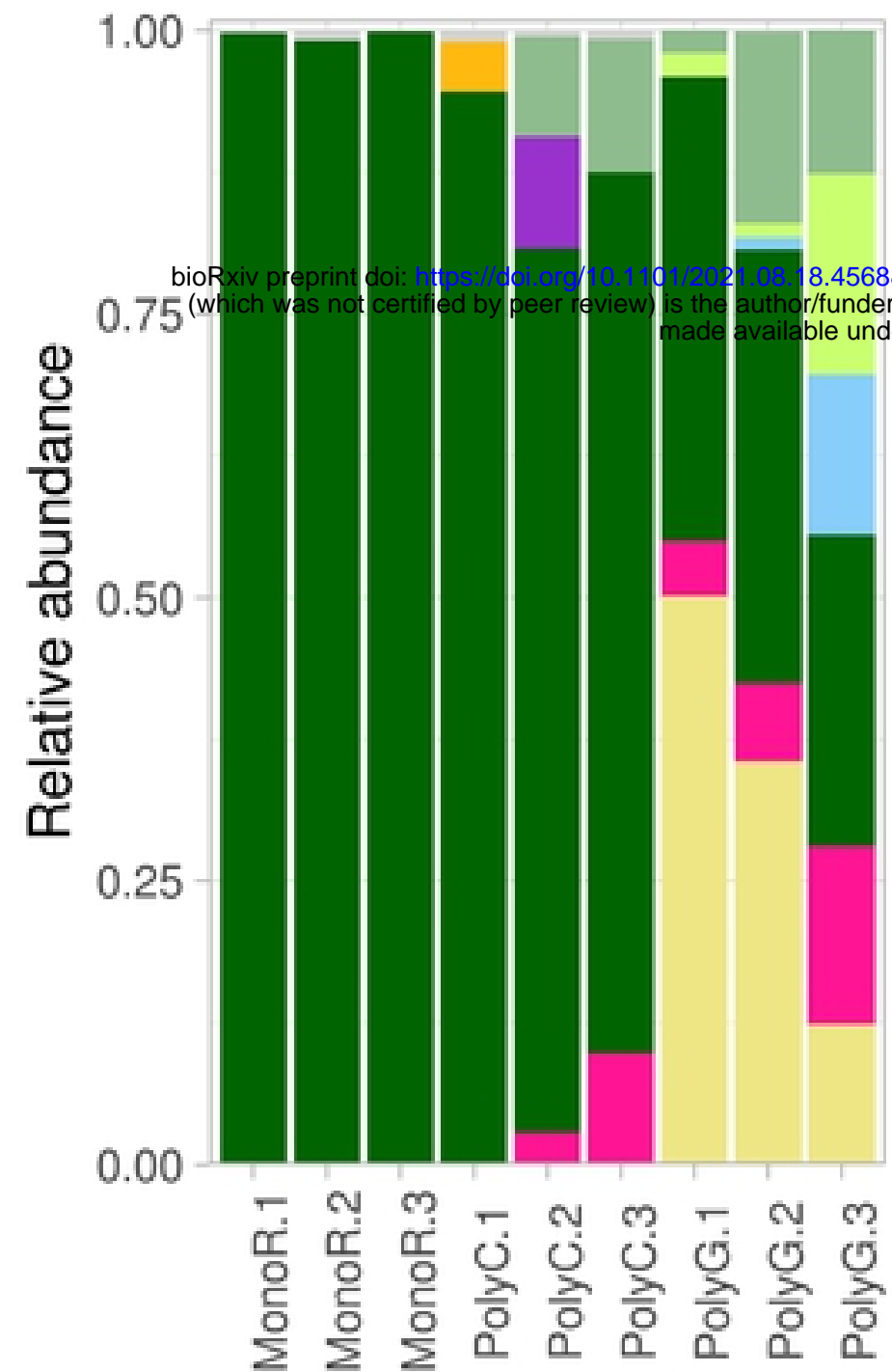

\section{B. Bacterial diversity}

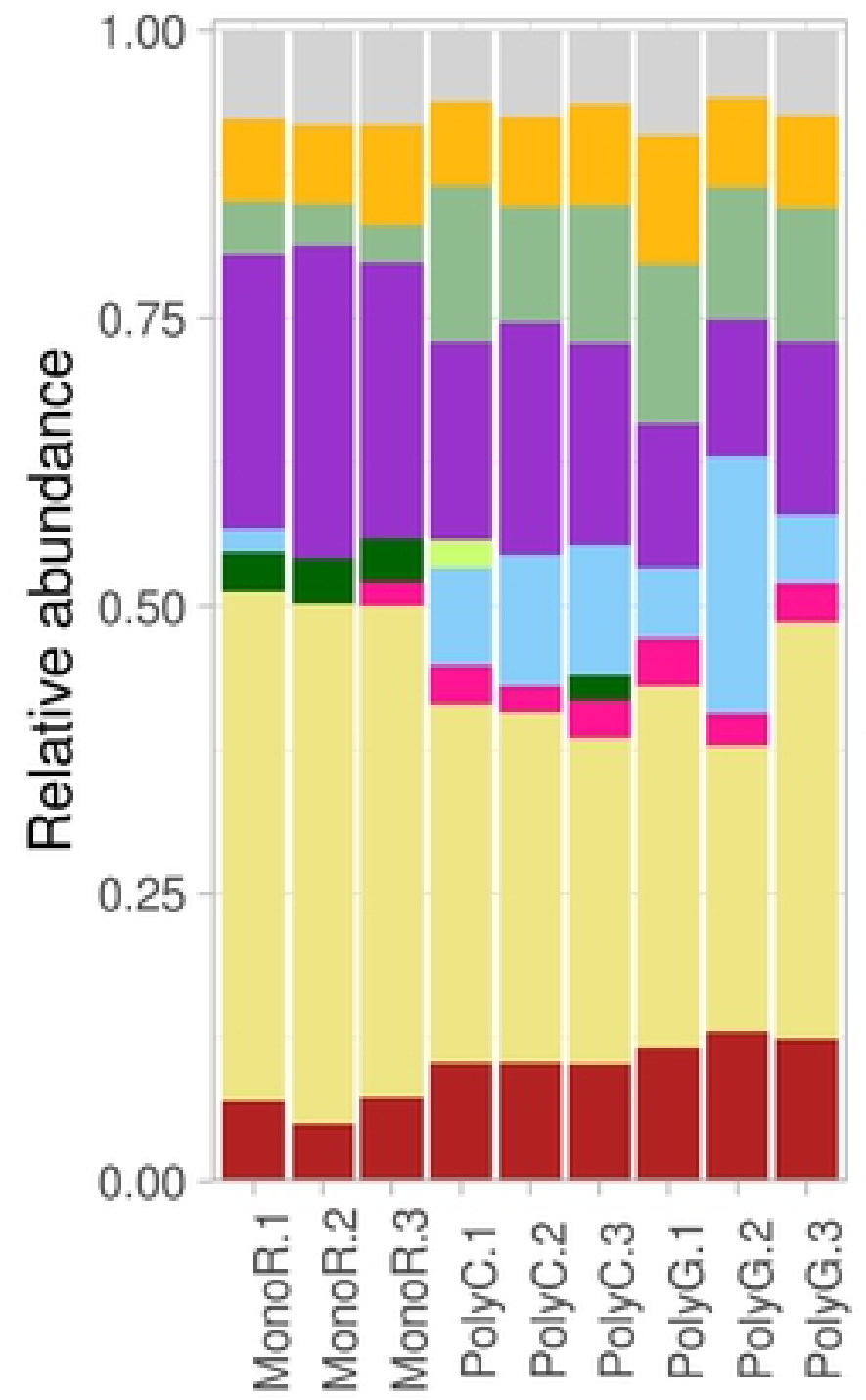

Family

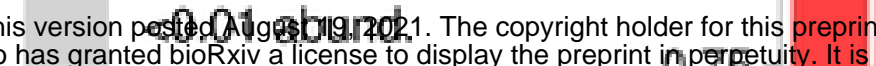
.

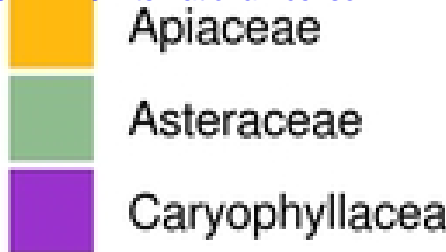

Fabaceae

Plantaginaceae

Poaceae

Rosaceae

Rubiaceae
Phylum

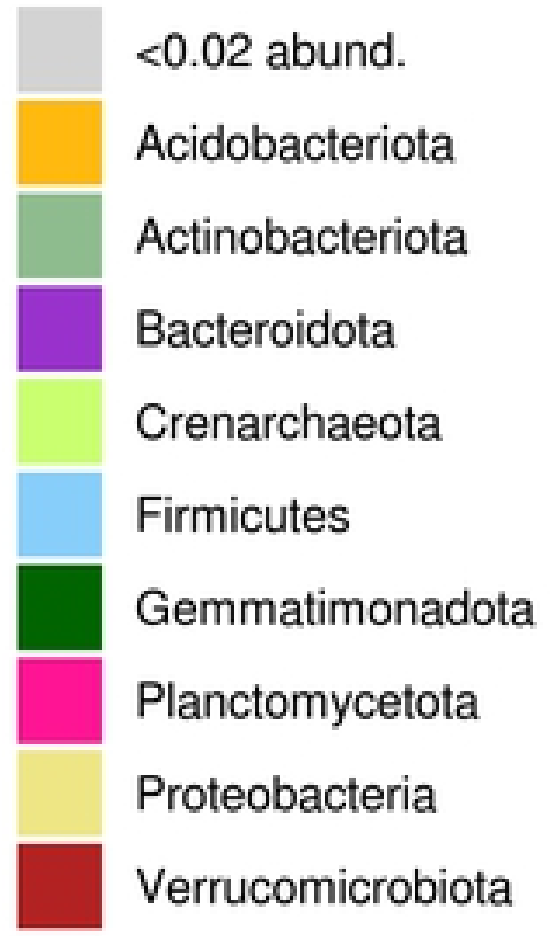

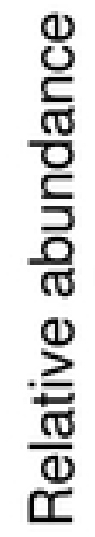

0.00

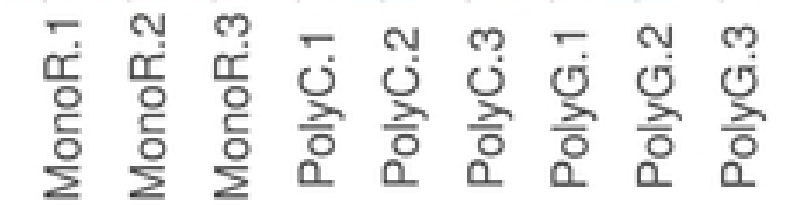

$<0.05$ abund.

Achillea

Alopecurus

Dactylis

Elymus

Festuca

Fragaria

Galium

Lolium

Poa

Secale

Stellaria

Taraxacum

Veronica

Vicia

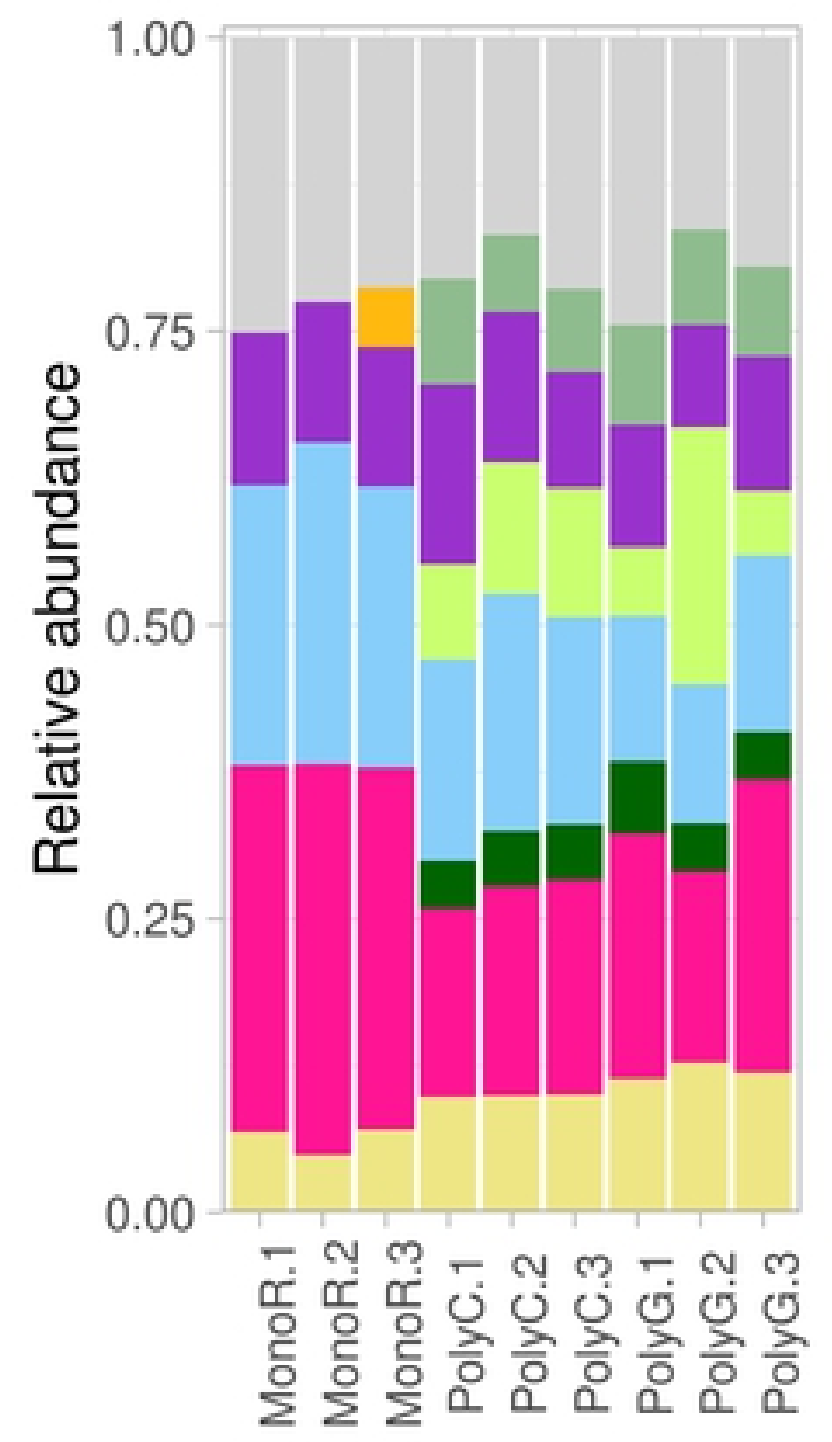

Class

$<0.04$ abund.

Acidobacteriae

Actinobacteria

Alphaproteobacteria

Bacilli

Bacteroidia

Blastocatellia

Gammaproteobacteria

Verrucomicrobiae 